\title{
Real-World Experience with Etanercept Therapy and the Switching Pattern among Korean Patients with Psoriasis after Withdrawal of Etanercept
}

\author{
Chong Won Choi, Ji Su Lee, Da-Ae Yu, Bo Ri Kim, Sang Woong Youn \\ Department of Dermatology, Seoul National University Bundang Hospital, Seoul National University College of Medicine, Seongnam, \\ Korea
}

Background: The efficacy and safety of etanercept in the treatment of psoriasis has been proven, and the drug was approved for the treatment of moderate to severe psoriasis. However, there have been few studies that have presented real-world data focused on concomitant treatment during etanercept treatment, and the switching pattern after discontinuation of etanercept. Objective: To reveal the real-world treatment pattern of etanercept-based psoriasis treatment and to investigate the switching pattern after withdrawal of etanercept. Methods: We enrolled 66 patients with psoriasis who were treated with etanercept. We collected data regarding the demographic characteristics of the patients, etanercept treatment schedules, and other treatments administered during the etanercept treatment period. We also investigated the treatment pattern after the discontinuation of etanercept with emphasis on the drug-free interval and the administered treatment modalities. Results: The mean treatment duration was $22.7 \pm 26.1$ months and the mean number of etanercept injections was $21.5 \pm 27.9$. Thirty-six patients were administered concomitant systemic medication

Received May 10, 2018, Revised August 15, 2018, Accepted for publication August 17, 2018

Corresponding author: Sang Woong Youn, Department of Dermatology, Seoul National University Bundang Hospital, Seoul National University College of Medicine, 82 Gumi-ro 173beon-gil, Bundang-gu, Seongnam 13620, Korea. Tel: 82-31-787-7319, Fax: 82-31-787-4058, E-mail: swyoun@snu.ac.kr

ORCID: https://orcid.org/0000-0002-5602-3530

This is an Open Access article distributed under the terms of the Creative Commons Attribution Non-Commercial License (http://creativecommons. org/licenses/by-nc/4.0) which permits unrestricted non-commercial use, distribution, and reproduction in any medium, provided the original work is properly cited.

Copyright $₫$ The Korean Dermatological Association and The Korean Society for Investigative Dermatology or phototherapy. After discontinuation of etanercept, 54 patients were followed up and 34 of these patients were administered other systemic medication or phototherapy; phototherapy and cyclosporine was the most commonly administered treatment modality and $27.4 \%$ of treatments used biologics. Conclusion: The treatment schedule for etanercept was modified according to the severity of psoriasis and concomitant treatment was administered to improve the effectiveness of treatment in the patients enrolled in the study. We also found that most patients required other treatment modalities to control psoriasis during the period of etanercept treatment. (Ann Dermatol 31(1) $44 \sim 50,2019$ )

\section{-Keywords-}

Psoriasis, Etanercept, Treatment, Combination, Switching pattern

\section{INTRODUCTION}

Psoriasis is a chronic inflammatory skin disorder that is associated with a significant decline in the quality of life of patients ${ }^{1}$. The chronic and recurrent nature of psoriasis often requires long-term continuous treatment ${ }^{2,3}$. As the understanding of the role of the immune mechanism in psoriasis has increased, new biologics have been introduced in the treatment ${ }^{1,4}$. In Korea, infliximab, etanercept, adalimumab, ustekinumab, and secukinumab are available for the treatment of moderate to severe psoriasis. Etanercept is a fully human tumor necrosis factor (TNF)- $\alpha$ receptor protein that competitively inhibits the binding of TNF- $\alpha$ to the cell surface receptors, thereby preventing the activation of the TNF- $\alpha$-mediated inflammatory cascade in patients with psoriasis ${ }^{1,4}$. The efficacy and safety of eta- 
nercept has been proven in the treatment of psoriasis, and it has been approved for the treatment of moderate to severe psoriasis since 2004 in Korea ${ }^{1,4}$. The utility of etanercept as an effective form of treatment for moderate to severe psoriasis has been investigated through various clinical trials; however, there have been few studies presenting real-world data focused on the switching pattern of treatment modalities after the discontinuation of etanercept. Previously, we reported the effectiveness of a low-dose etanercept therapy combined with systemic medication or phototherapy in Asian patients with non-severe psoriasis in comparison to that in a Western population ${ }^{1}$. In addition, other studies have reported the effectiveness of an intermittent etanercept treatment or a combination treatment with etanercept and acitretin for patients with plaque-type psoriasis $^{2,4}$. Based on these previous studies, it can be inferred that etanercept is used with modified treatment regimens and in combination with other treatment modalities. However, there have been very few studies focused on concomitant systemic medication or phototherapy. Therefore, we investigated the effectiveness of concomitant treatment during etanercept treatment.

Newly introduced biologics have been reported to have better efficacy and the treatment schedule is simpler than what is used for etanercept-based treatment. Moreover, currently, etanercept is not frequently used for the treatment of psoriasis. A study on the pattern of etanercept treatment may reflect the need for treatment of patients with psoriasis. Such a study would allow us to anticipate the therapeutic pattern of newly developed biologics. Therefore, we performed an observational study to investigate the real-world experience of etanercept-based psoriasis treatment. This study is expected to reveal the essential elements of a good treatment modality for psoriasis.

\section{MATERIALS AND METHODS}

\section{Patients and data acquisition}

We enrolled 66 patients with psoriasis who were treated with etanercept between September 2004 and January 2016 at Seoul National University Bundang Hospital. Data on the demographic characteristics of the patients (comprising of information on age, sex, body mass index, comorbidity, duration of psoriasis, previous treatments for psoriasis, and biologics naivety), data related to the etanercept treatment (including information on other treatment modalities introduced during the treatment), and information regarding the treatment pattern after etanercept discontinuation (with emphasis on the drug-free interval and the administrated treatment modalities) were collected and analyzed to determine the characteristics and the real-world experience of etanercept treatment and the switching pattern after etanercept discontinuation. We enrolled 66 patients with psoriasis who were treated with etanercept between September 2004 and January 2016 at Seoul National University Bundang Hospital (Fig. 1).

\begin{tabular}{|c|c|}
\hline $\begin{array}{l}\text { Experience with etanercept treatment in real world } \\
\qquad(n=66)\end{array}$ & \\
\hline \multicolumn{2}{|c|}{ Characteristics of etanercept treatment } \\
\hline \multicolumn{2}{|c|}{$\begin{array}{l}\text { Total number of etanercept injection } \\
\text { Cumulative doses of etanercept (mg) }\end{array}$} \\
\hline \multicolumn{2}{|c|}{ Concomitant treatment during etanercept therapy } \\
\hline $\begin{array}{l}\text { Etanercept only } \\
\text { Concomitant systemic } \\
\text { Concomitant photothe }\end{array}$ & $\begin{array}{l}30(45.5 \%) \\
16(24.2 \%) \\
24(36.4 \%)\end{array}$ \\
\hline \multicolumn{2}{|l|}{$\begin{array}{l}\text { Switching pattern after discontinuation of etanercept } \\
\qquad(\mathrm{n}=54)\end{array}$} \\
\hline & $\begin{array}{l}\text { Characteristics of } 95 \text { treatments after discontinuation of etanercept } \\
\text { Duration up to starting retreatment } \\
\text { Treatment modalities for psoriasis treatment }\end{array}$ \\
\hline $\begin{array}{l}\text { Phototherapy } \\
\text { Cyclosporine A } \\
\text { Methotrexate } \\
\text { Acitretin } \\
\text { Ustekinumab } \\
\text { Adalimumab } \\
\text { Guselkumab }\end{array}$ & $\begin{array}{c}24(44.4 \%) \\
24(44.4 \%) \\
15(27.8 \%) \\
6(11.1 \%) \\
21(38.9 \%) \\
2(3.7 \%) \\
3(5.6 \%)\end{array}$ \\
\hline
\end{tabular}

Fig. 1. Study design and summary of results. 
The treatment regimen of etanercept in most patients enrolled in this study was modified from the standard regimen. The treatment regimen for the enrolled patients was initiated as a twice-weekly injection of etanercept. The dose and interval of etanercept injection was changed according to the patient's response to the treatment. The majority of patients were treated intermittently and the intervals between the injections were not consistent. Because some of the enrolled patients used etanercept intermittently, we defined the duration of the etanercept treatment period as between the date of first injection of etanercept and the date of last injection. Furthermore, concomitant treatment was defined as the use of other psoriasis treatment modalities, which were administered during the etanercept treatment period.

This study was approved by the institutional review board of Seoul National University Bundang Hospital (IRB number: B-1603/340-105).

\section{Statistical analysis}

Statistical analyses were performed using the Student t-test for comparing continuous variables. The chi-square test and Fisher's exact test were used for the analysis of categorical variables. Results were expressed as the mean \pm standard deviation (SD). We considered a $p$-value of $<0.05$ as statistically significant. Statistical analysis was performed using the IBM SPSS Statistics ver. 24.0 (IBM Co., Armonk, NY, USA).

\section{RESULTS}

\section{Characteristics of patients and etanercept treatment}

The mean \pm SD age and duration of psoriasis at the initiation of etanercept treatment for the 66 enrolled patients with psoriasis was $52.8 \pm 15.4$ years and $13.3 \pm 12.2$ years, respectively (Table 1$)$. Among these patients, chronic liver disease was found in 7 (10.6\%) patients and was the most common comorbidity, followed by hyperlipidemia (5 [7.6\%] patients) and hypertension (5 [7.6\%] patients). Before undergoing etanercept treatment, $40(60.6 \%)$ patients had undergone phototherapy, and 13 (19.7\%) and 12 (18.2\%) patients had received acitretin and cyclosporine for the treatment of psoriasis, respectively. Sixty-four (97.0\%) patients were biologics-naïve, and the remaining two (3\%) had used biologics: one patients had been administered ustekinumab and adalimumab, while the other had been administered efalizumab. Among the 66 patients with psoriasis, etanercept was first injected in November 2004 and the last injection was performed in July 2015.

The mean duration of the etanercept treatment period was $22.7 \pm 26.1$ months and the mean number of etanercept
Table 1. Baseline demographics and characteristics of patients $(\mathrm{n}=66)$

\begin{tabular}{|c|c|}
\hline Characteristics & Value \\
\hline Age when starting etanercept $(\mathrm{yr})$ & $52.8 \pm 15.4$ \\
\hline \multicolumn{2}{|l|}{ Sex } \\
\hline Male & $40(60.6)$ \\
\hline Female & $26(39.4)$ \\
\hline Body mass index $\left(\mathrm{kg} / \mathrm{m}^{2}\right)^{*}$ & $25.3 \pm 3.8$ \\
\hline \multicolumn{2}{|l|}{ Co-morbidities } \\
\hline Diabetes & $3(4.5)$ \\
\hline Hyperlipidemia & $5(7.6)$ \\
\hline Hypertension & $5(7.6)$ \\
\hline Chronic liver disease & $7(10.6)$ \\
\hline Latent tuberculosis & $2(3.0)$ \\
\hline Chronic kidney disease & $2(3.0)$ \\
\hline Duration of psoriasis $(\mathrm{yr})^{\dagger}$ & $13.3 \pm 12.2$ \\
\hline \multicolumn{2}{|l|}{ Previous therapy } \\
\hline Topical agents & $66(100.0)$ \\
\hline Phototherapy & $40(60.6)$ \\
\hline \multicolumn{2}{|l|}{ Systemic medication } \\
\hline Cyclosporine A & $12(18.2)$ \\
\hline Methotrexate & $3(4.5)$ \\
\hline Acitretin & $13(19.7)$ \\
\hline \multicolumn{2}{|l|}{ Biologics } \\
\hline Efalizumab & $1(1.5)$ \\
\hline Adalimumab & $1(1.5)$ \\
\hline Ustekinumab & $1(1.5)$ \\
\hline Treatment duration of etanercept therapy (mo) & $22.7 \pm 26.1$ \\
\hline Total number of etanercept injection (range) & $\begin{array}{l}21.5 \pm 27.9 \\
(1 \sim 166)\end{array}$ \\
\hline Cumulative doses of etanercept (mg) & $543.2 \pm 697.0$ \\
\hline $\begin{array}{l}\text { Concomitant systemic medication during } \\
\text { etanercept therapy }\end{array}$ & $16(24.2)$ \\
\hline Cyclosporine A & $13(19.7)$ \\
\hline Methotrexate & $6(9.1)$ \\
\hline Acitretin & $5(7.6)$ \\
\hline $\begin{array}{l}\text { Concomitant phototherapy during etanercept } \\
\text { therapy }\end{array}$ & $24(36.4)$ \\
\hline \multicolumn{2}{|l|}{ Reason for etanercept withdrawal } \\
\hline Withdrawal after sufficient improvement & $23(34.8)$ \\
\hline Adverse reactions & $3(4.5)$ \\
\hline Treatment failure & $14(21.2)$ \\
\hline Burden of expenses & $3(4.5)$ \\
\hline Patient preference & $3(4.5)$ \\
\hline Switch to ustekinumab & $3(4.5)$ \\
\hline Loss to follow-up & $17(25.8)$ \\
\hline
\end{tabular}

Values are presented as mean \pm standard deviation or number $(\%) . *$ Forty-three patients with unrecorded weight or height were omitted from the calculation. ${ }^{\dagger}$ Two patients with unknown time of psoriasis onset were omitted from the calculation.

injections and the mean cumulative dose was $21.5 \pm 27.9$ and $543.2 \pm 697.0 \mathrm{mg}$ (Table 1). Among the 66 patients, 36 patients were administered concomitant systemic medication or phototherapy during the etanercept treatment period. At the time of etanercept initiation, two and 11 pa- 
Table 2. Characteristics of initial treatment after the withdrawal of etanercept among the patients who continuously treated at our hospital $(\mathrm{n}=34)^{*}$

\begin{tabular}{lc}
\multicolumn{1}{c}{ Characteristics } & Value \\
\hline Time until the new treatment initiation (mo) & $15.5 \pm 35.6$ \\
Treatment modality & $5(14.7)$ \\
Phototherapy $^{\dagger}$ & \\
Systemic medication & $14(41.2)$ \\
Cyclosporine A $^{\text {Methotrexate }}$ & $2(5.9)$ \\
Acitretin $^{\dagger}$ & $4(11.8)$ \\
Biologic agents & \\
Ustekinumab & $9(26.5)$ \\
Adalimumab & $1(2.9)$ \\
\hline
\end{tabular}

Values are presented as mean \pm standard deviation or number (\%). ${ }^{*}$ Of the 66 patients, 34 switched their treatment modality after the discontinuation of etanercept. ${ }^{\dagger}$ There was one patient who was retreated with simultaneous acitretin and phototherapy.

tients were already on cyclosporine treatment and phototherapy, respectively. For the rest of these patients, however, concomitant systemic medication or phototherapy was initiated after the initiation of etanercept treatment. Among them, 14 patients were treated with cyclosporine, 6 with methotrexate, 5 with acitretin, and 24 with phototherapy during the etanercept treatment period. After an average of $4.6 \pm 7.5$ months of etanercept treatment, concomitant systemic medication or phototherapy was initiated for 23 patients for psoriasis treatment. At the time of etanercept discontinuation, a total of 13 patients were on concomitant systemic medication or phototherapy owing to the loss of etanercept effectiveness. Among them, 4 patients were treated with cyclosporine, 3 with methotrexate, and 1 with acitretin. In addition, 7 patients were on phototherapy when etanercept treatment was discontinued. Two patients were administered a combination therapy of cyclosporine and phototherapy. There were no adverse events in those patients despite the overlap of immunosuppressive therapy (etanercept with cyclosporine, or etanercept with phototherapy).

\section{Characteristics of treatment after the discontinuation of etanercept}

After discontinuation of etanercept, we followed up with 54 patients during the study period. The other 12 patients were lost to follow-up. Of the 54 patients, 34 patients were continuously treated at out hospital and remaining 20 were treated at other hospitals after discontinuation of etanercept for a while and came back to our hospital during the study period. To analyze the switching pattern after discontinuation of etanercept, we summarized the ini-
Table 3. Characteristics of 95 treatments after the discontinuation of etanercept $(n=54)^{*}$

\begin{tabular}{lc}
\hline \multicolumn{1}{c}{ Characteristics } & Value \\
\hline $\begin{array}{l}\text { Duration up to starting retreatment (mo) } \\
\text { Retreatment modality }\end{array}$ & $27.5 \pm 52.8$ \\
Topical agents & $54(100)$ \\
Phototherapy & $24(44.4)$ \\
Systemic medication & \\
Cyclosporine A & $24(44.4)$ \\
Methotrexate & $15(27.8)$ \\
Acitretin & $6(11.1)$ \\
Biologic agents & \\
Ustekinumab & $21(38.9)$ \\
Adalimumab & $2(3.7)$ \\
Guselkumab & $3(5.6)$ \\
\hline
\end{tabular}

Values are presented as mean \pm standard deviation or number $(\%)$. *Twelve of 66 patients who discontinued etanercept treatment were lost during follow-up. There were some patients retreated with more than two modalities that are listed in the table.

tial treatment of 34 patients who continuously treated at our hospital (Table 2). The mean time to initiation of new treatment modalities among 34 patients was $15.5 \pm 35.6$ months. Cyclosporine treatment was the most commonly administered treatment modality $(14 ; 41.2 \%)$, followed by ustekinumab $(9 ; 26.5 \%)$ and phototherapy $(5 ; 14.7 \%)$. During the follow-up period, we administered a total of 95 treatments in 54 patients (Table 3). Among the administered treatments, phototherapy $(24 ; 44.4 \%)$ and cyclosporine $(24 ; 44.4 \%)$ were the most commonly administered treatment modalities, followed by ustekinumab $(21 ; 38.9 \%)$.

The treatment for $26(27.4 \%)$ patients after etanercept discontinuation involved the use of biologics. To determine the factors that influence the patient's decision of using biologics for the treatment of psoriasis, we compared the epidemiologic characteristics of the patients and the characteristics of the etanercept treatment between those who chose biologics after etanercept discontinuation and those who did not (Table 4). Between the two groups, the proportion of patients who underwent phototherapy before etanercept was higher among those who chose biologics after the discontinuation of etanercept $(p=0.046)$.

\section{DISCUSSION}

Etanercept is a fully human TNF- $\alpha$ receptor protein that competitively inhibits the TNF- $\alpha$-mediated inflammatory cascade in patients with psoriasis ${ }^{1,4}$. The common regimen of etanercept for chronic plaque-type psoriasis in Korea involves a biweekly injection of $25 \mathrm{mg}$ of etanercept ${ }^{1,2}$. In 
Table 4. Comparison between the patients who chose biologics after etanercept discontinuation and those who did not

\begin{tabular}{|c|c|c|c|}
\hline Characteristics & Non-biologics $(n=28)$ & Biologics $(n=26)$ & $p$-value \\
\hline Age when starting etanercept (yr) & $45.4 \pm 15.5$ & $43.6 \pm 16.6$ & 0.398 \\
\hline Sex & & & 0.377 \\
\hline Female & $13(46.4)$ & $9(34.6)$ & \\
\hline Male & 15 (53.6) & $17(65.4)$ & \\
\hline Body mass index $\left(\mathrm{kg} / \mathrm{m}^{2}\right)^{*}$ & $25.4 \pm 4.1$ & $25.3 \pm 3.8$ & 0.960 \\
\hline \multicolumn{4}{|l|}{ Comorbidities } \\
\hline Diabetes & $2(7.1)$ & $1(3.8)$ & 1.000 \\
\hline Hyperlipidemia & $4(14.3)$ & $1(3.8)$ & 0.353 \\
\hline Hypertension & $3(10.7)$ & $2(7.7)$ & 1.000 \\
\hline Chronic hepatopathy & $4(14.3)$ & $3(11.5)$ & 1.000 \\
\hline Latent tuberculosis & $2(7.1)$ & $0(0.0)$ & 0.491 \\
\hline Renal insufficiency & $1(3.6)$ & $0(0.0)$ & 1.000 \\
\hline Duration of psoriasis (mo) & $147.7 \pm 141.6$ & $191.8 \pm 164.3$ & 0.305 \\
\hline \multicolumn{4}{|l|}{ Previous therapy } \\
\hline Phototherapy & $13(46.4)$ & $19(73.1)$ & 0.046 \\
\hline Cyclosprorine & $5(17.9)$ & $6(23.1)$ & 0.634 \\
\hline Methotraxate & $1(3.6)$ & $2(7.7)$ & 0.604 \\
\hline Acitretin & $8(28.6)$ & $4(15.4)$ & 0.244 \\
\hline Biologic agents & $0(0.0)$ & $2(7.7)$ & 0.227 \\
\hline Treatment duration (mo) & $21.7 \pm 28.4$ & $25.9 \pm 24.4$ & 0.563 \\
\hline Total number of etanercept injection & $16.9 \pm 13.4$ & $25.7 \pm 29.5$ & 0.169 \\
\hline Cumulative doses (mg) & $424.1 \pm 334.5$ & $653.8 \pm 733.6$ & 0.153 \\
\hline Concomitant treatment & $18(64.3)$ & $15(57.7)$ & 0.781 \\
\hline Duration up to starting retreatment (mo) & $7.7 \pm 23.5$ & $10.6 \pm 17.9$ & 0.771 \\
\hline
\end{tabular}

Values are presented as mean \pm standard deviation or number (\%). *Five patients in the non-biologics and 10 in the biologics group with unrecorded weight or height were omitted from the calculation. Statistics were analyzed using the Chi square test, Fisher's exact test, and independent t-test.

case of poor response, the dose of etanercept can be increased up to $50 \mathrm{mg}^{2}$. However, modified etanercept treatment regimens, such as low-dose etanercept therapy or combination treatment involving etanercept and other systemic medications have been proposed to improve the effectiveness or lower the cost of etanercept treatment ${ }^{1,2}$. Previously, we suggested that a low-dose etanercept therapy combined with conventional treatment modalities could be an alternative and less-expensive treatment for the patients with psoriasis who do not responding well to conventional etanercept treatment ${ }^{1}$. In this study, we found that systemic medication or phototherapy were administered during the etanercept treatment period when the effectiveness of the etanercept treatment was not sufficient. In addition, we also found that systemic medication or phototherapy were used between the intermittent etanercept treatments. Among the 36 patients $(54.5 \%)$ who were administered systemic medication or phototherapy during the etanercept treatment period, etanercept was initiated during the systemic medication or phototherapy in 13 patients. In contrast, concomitant systemic medication or phototherapy was initiated for the rest after initiation of etanercept treatment. The time interval between the initiation of etanercept treatment and the initiation of concomitant systemic medication or phototherapy was $4.6 \pm 7.5$ months in 23 patients. The time interval suggested that single therapy with etanercept is unable to control the activity of psoriasis. In this study, we again confirmed that the etanercept therapy combined with conventional treatment modalities could be used effectively in Korean patients with psoriasis.

Since psoriasis involves the chronic relapsing inflammation of the skin, the combination treatment and rotational approach was introduced to achieve sustained disease control without the development of adverse events ${ }^{3,5}$. In this study, we found that a total of 36 patients were treated with concomitant systemic medication or phototherapy as combinational or rotational treatment during the etanercept treatment period. Among them, phototherapy was the most frequently administered treatment modality during the etanercept treatment period, followed by cyclosporine. This result is slightly different from what is reported in previous studies. Busard et al. ${ }^{6}$ investigated the conventional systemic agents or phototherapy combined with biologics and found that methotrexate was most frequently used compound. However, this study only ana- 
lyzed the concomitant combined treatment during the use of biologics, whereas we investigated the combined treatment with etanercept and the rotational treatment between intermittent etanercept treatments. Considering the profuse experience in the combined use of methotrexate with biologics ${ }^{6,7}$, the results of previous studies and the results from ours will justifiably have some differences.

In a previous study performed in Germany, the mean drug-free intervals after etanercept discontinuation was 12.9 weeks $^{4}$. This study also stated that $37.3 \%$ of patients who discontinued etanercept treatment switched to another anti-TNF agent ${ }^{4}$. In this study, we investigated the treatment pattern after etanercept discontinuation and analyzed the switching pattern. Among the enrolled patients, 34 patients initiated another treatment for psoriasis after $15.5 \pm 35.6$ months and 10 of these patients $(29.4 \%)$ chose other biologics. Compared to the previous study, this longer drug-free interval can be a result of the lower severity of psoriasis in Asian patients compared to Caucasians. Lastly, we have followed up 54 patients with psoriasis and found that $26(48.1 \%)$ used biologics during the follow-up period. Among the other biologics used during the follow-up period, ustekinumab was the most commonly administered. To determine the factors that influence patients with psoriasis to choose biologics, we performed additional analysis and found that the proportion of patients who experienced phototherapy before starting etanercept was higher in the patients choosing other biologics after etanercept discontinuation. It may be inferred that the concern regarding the possibility of long-term adverse events resulting from the continued use of systemic medications might influence the choice of using phototherapy or biologics.

In choosing the treatment modalities, the severity of psoriasis can be a determining factor. As our study was a retrospective study using data from medical chart in real-world, assessing the Psoriasis Area and Severity Index (PASI) score was not performed in every visit. For this reason, we could not retrieve enough PASI scores for analysis and we are not able to analyze the effect of severity of psoriasis on the treatment pattern or switching pattern of psoriasis treatment.

In conclusion, for Asian patients with a milder form of psoriasis in comparison to Caucasians patients, etanercept therapy with concomitant systemic medication or phototherapy can be an effective treatment modality. Considering the introduction of new biologics with superior therapeutic efficacy into the treatment regimen of psoriasis ${ }^{8,9}$, the use of biologics in psoriasis treatment is expected to increase further and the combinational and rotational treatment with new biologics and conventional systemic medication or phototherapy will be introduced to improve the effectiveness of psoriasis treatment and reduce the economic burden associated with psoriasis treatment.

\section{CONFLICTS OF INTEREST}

Dr. Youn has served as an advisor, received speaker honoraria, and participated in clinical trials for AbbVie, CKD-pharma, Elli-Lilly, Janssen, and Novartis. He participated in a clinical trial for Kyowa-Kirin. He also served as an advisor for Celgene.

\section{ORCID}

Chong Won Choi, https://orcid.org/0000-0001-9994-8819

Ji Su Lee, https://orcid.org/0000-0003-0207-2107

Da-Ae Yu, https://orcid.org/0000-0001-8158-9766

Bo Ri Kim, https://orcid.org/0000-0002-2223-1606

Sang Woong Youn, https://orcid.org/0000-0002-5602-3530

\section{REFERENCES}

1. Na Jl, Kim JH, Park KC, Youn SW. Low-dose etanercept therapy in moderate to severe psoriasis in Korean. J Dermatol 2008;35:484-490.

2. Lee JH, Youn JI, Kim TY, Choi JH, Park CJ, Choe YB, et al. A multicenter, randomized, open-label pilot trial assessing the efficacy and safety of etanercept $50 \mathrm{mg}$ twice weekly followed by etanercept $25 \mathrm{mg}$ twice weekly, the combination of etanercept $25 \mathrm{mg}$ twice weekly and acitretin, and acitretin alone in patients with moderate to severe psoriasis. BMC Dermatol 2016;16:11.

3. Choi CW, Kim BR, Ohn J, Youn SW. The advantage of cyclosporine $\mathrm{A}$ and methotrexate rotational therapy in long-term systemic treatment for chronic plaque psoriasis in a real world practice. Ann Dermatol 2017;29:55-60.

4. Luger T, Schopf RE, Schwanke A, Langhammer S, Meng T, Löschmann PA. An observational study to evaluate the long-term outcomes of treatment with etanercept in patients with plaque-type psoriasis. J Eur Acad Dermatol Venereol 2016;30:1730-1741.

5. Lebwohl M, Menter A, Koo J, Feldman SR. Combination therapy to treat moderate to severe psoriasis. J Am Acad Dermatol 2004;50:416-430.

6. Busard $\mathrm{Cl}$, Cohen AD, Wolf P, Gkalpakiotis S, Cazzaniga S, Stern RS, et al. Biologics combined with conventional systemic agents or phototherapy for the treatment of psoriasis: real-life data from PSONET registries. J Eur Acad Dermatol Venereol 2018;32:245-253.

7. Zweegers J, Otero ME, van den Reek JM, van Lümig PP, Driessen RJ, Kievit W, et al. Effectiveness of biologic and conventional systemic therapies in adults with chronic plaque psoriasis in daily practice: a systematic review. Acta Derm Venereol 2016;96:453-458.

8. Kimball AB, Papp KA, Wasfi Y, Chan D, Bissonnette R, 
Sofen $\mathrm{H}$, et al. Long-term efficacy of ustekinumab in patients with moderate-to-severe psoriasis treated for up to 5 years in the PHOENIX 1 study. J Eur Acad Dermatol Venereol 2013;27:1535-1545.
9. Vergou T, Moustou AE, Antoniou C. Five-year experience with Ustekinumab for psoriasis: real-life data of a single centre. J Eur Acad Dermatol Venereol 2017;31:e40-41. 